Dermatologische Zeitschrift. 1935;70:I-IV

\title{
Contents, Vol. 70, 1935
}

\section{Dermatologiseh $\theta$ Zeitsehrift}

Berichtsorgan der Berliner Dermatologischen Gesellschaft Begründet von O. LASSAR Unter Mitwirkung von Saînz de Aja-Madrid; J. Almkvist-Stockholm; Ed. Arní đg-Hamburg; F. Bering-Köln; H. Boas-Kopenhagen; J. S. Covísa-Madrid; C Cronquist-Malmö; J. Darier-Paris; U. Ebbecke-Bonn; R. Frühwald-Chemnitz; W. Gernerich-Kiel; C. Grouven-Halle; 0. GrützBonn; R. Haber-maสn - Hamburg; L. Hauck-Erlangen; W. Meuck-München; E. HofmannKassel; F. Jahnel-München; A. Jesînek-Gießen; A. Jordan-Moskau; P. Keller-Aachen; W. KerlWien; W.KolIe-Frankfurt; L. Kumer-Innsbruck; v. Leeuwen Utrecht; P.Li $\pi$ ser-Tübingen; E. G. Graham Little-London; H. Lohe-Berlin; W. Lutz-Basel; S. Matsumoto-Kyoto; J. Mayr-Münster; A. Memmes-heimer-Essen; H. Meyer-Bremen; G. Mîescher- Zurich; C. Motcorps-München; P. Mulzer-Hamburg; 0. Naegeli-Bern; L. Nékám-Budapest; G. Nobl-Wien; A. Pasini-Mailand; G. Th. Photinos-Athen; R. Polland-Graz; C. Rasch-Kopenhagen; E. Ríecke-Göttingen; H. RitterHamburg; G. Rost-Berlin; A. Ruete-Marburg; G. Scherber-Wien; W. I. Schmidt-Gießen; W. Scholtz-Königsberg; W. Schonfeld-Greifswald; H. Th. Schreus-Düsseldorf; H. W. Siemens-

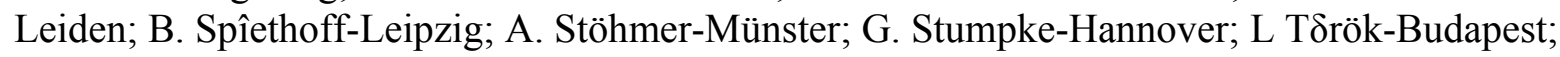
M. Truffi-Padua; P. Uhlenhuth-Freiburg; E. Urbach-Wien; H. Vorner-Leipzig; J. WertherDresden; Ch. J. White - Boston; K. Zieler-Würzburg; L. von Zumbusch - München herausgegeben in Gemeinschaft mit

L. Ârzt A. Buschke W. Trieboes

Wien Berlin Berlin
K. HerxheîmerV. Klingmûller
E. Zurhelle

Frankfurt Kiel Groningen

von Erich Hoffmann Bonn

Band 70

BERLIN 1935

VERLAG VON S. KARGER

KARLSTRASSE 39

Mit zahlreichen Abbildungen im Text und Register zu Band 66-70

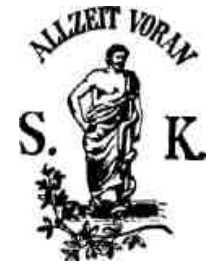

Alle Rechte vorbehalten

Pierersch $\theta$ Hofbuchdruckerei Stephan Geibel \& Co., Ältenburg (Thür.).

Inhaltsverzeichnis.

Or $1 / 8$ inalarbeiten.

Seit $\beta$ 
Akowbjan, A., Temporãre psychische Storung nach Salvarsanînjektion ... 31 Boas, Haralä, Kann eine positive Seroreaktion bei Syphilis ohne Behand-

lung negativ werden? 318

Carrie, C, Die Ursache d $7 / 8$ r Porphyrin-Fluoreszenz in der Mundhõhle und

auf der Haut 189

Cordes, W., siehe W. Engelhardt.

Epstein, Stephan, Beitrag zur staphylogenen Impetigo contagiosa .... 328

Engelharät, W. und W. Cordes, Der Einfluß der Kriegs- und Nachkriegs-

zeit auf Psoriasis und Hauttuberkulose 321

Földváń, F., Untersuchungen fiber Komplementbindung bei Pilzkrankheiten 260 Frohn, W., und II. 0. Loos, Ober doppelte Lãngskantenbildung der Nagel-

platte (Doppelkantennãgel) 76

Hoffmann, Erich, Zur 25jährigen Wiederkehr der Einführung des Ehr-

lich'schen Salvarsans in die Syphilisbehandlung 309

Hruszek, H., Über den Einfluß der Nährbodenmenge, des „Lichtes” und

der verimpften „Keimzahl” auf das Wachstum von Pilzen 197

- $\quad$ Pilzkulturen auf Gurkenschnitten , ein neuer natürlicher PПznährboden

fur Primär- und Passagekulturen 341

John, Ferdinand, Erfahrungen mit der Komplementbindungsreaktion bei

Gonorrhoe 19

Jordan, A., und A. Tarabuchin, Über Cheilitis exfoliativa und glandularis 249 Lewin, E. M., I.

M. Lewin, M. M. Lewin und M. S. Babinowitsch, Ober

intrakutane Reaktion mit Staphylotoxin bei Staphylodermie 82

Über die inhibitorische Wirku $\pi \mathrm{g}$ des Antivirus und fiber lokale

Antivirustherapie bei Furunkulose 202

Leivin, I. M., Über den Zusammenhang zwischen Zoster- und Varizellenvirus 346

- $\quad$ siehe E. M. Lewin.

Leivin, M. M., siehe Lewin, E. M.

Lomhoït, Sven, D., Über die Behandlung des Boeckschen Sarkoids mit

Antileprol 57

Loos, H. 0., siehe W. Frohn.

Memmesheimer, Alois M., Zur Entstehung und Behandlung des Ekzems vom

Standpunkt der Herdinfektionslehre 1

Papendieck, A., Über Vorteile von Pufferwirkungen bei der Ekzembehandlung 269

- Lipolimentum purum als Firnistinktur und physiologischer Grundstoff

für Hautfirnispi $\pi$ selungen 266

Piepmeyer, Erich, Über die Beziehung zwischen vitalen Kationenver-

schiebungen und Erregbarkeitslage des Hautorganes 133

Babinowitsch, M. 8., siehe E. M. Lewin.

Schaar, P. I. van der, Гramboesieimpfungen auf Paralytiker 185

Schreus, H. Th., Prinzip und Leistung der spezífisch sensibilisierten Wasser-

mannreaktion (W.S.R.) 121

Steffen, Walter, Behandlung der Zervix-Gonorrhoe mit Flavadin 194

Tarabuchin, A., siehe A. Jordan.

Vleugels-Schutter, G. J. N., Der Wert graphischer Darstellungen für die

Beurteilung der Behandlungsresultate bei Gonorrhoe $\quad 142$ 
IV Inhaltsverzeichnis.

Seite

Wagner, B., Über die Verweildauer von Sypliiiisspirocliäten im Blutegel 11

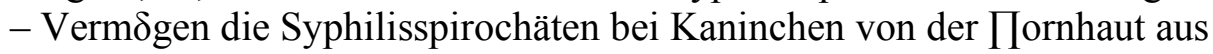

ins Gehirn einzudringen?

13

Willard, Viktor, Exantheme bei Sepsis mit besonderer Berücksichtigung

der Pneumokokken und Kolisepsis 271

Zípersson, I). A., Multiple Fibromatose der Haut nach Frostschaden . . .

Zoon, J. J., Über histologische Befunde bei Argyria cutis

125

Sammelreferate.

Strahlenheilkunde. Vo, Prof. Philipp Keller-Bad Aachen 34

Syphilis, ßeferat über Arbeiten des Jahres 1933. Von W. Schönfelä-Greifswsãd 87 Der Tripper.

Neue Ergebøisse aus dem Schrifttum vom Juli 1933 bis

Juni 1934. Von Prof. Dr. J. Hämel und Dr. II. G¹/8/er-Würzburg . . 151 Sexualerkrankungen

(Ulcus molle, Lymphogranuloma ing.), Prophylaxe und

Schwa Igerschaft. Von Hans Döllken·Königsberg i. Pr 207

Âllgemeine Dermatologie. I. Anatomie und Пistologie. (Herbst 1933 bis

Herbst 1934.) Von Max Walther-B\&d Homburg 283

Stoffwechsel. (Herbst 1933 bis Herbst 1934.) Von Privatdozent Dr. C. Mon-

corps-München 346

Zusammenfassende Arbeiten diagnostischen und therapeutischen Inhalts. Einige praktische

Diätformen bei der Ernährungsbehandlu $\pi$ g von Haut-

krankheiten. Von Prof. Dr. A. Marchionini und Dr. Fr. Böhníng-

Freiburg i. Br 44

Methoden zur Feststellung und Behandlung nutritiv-allergischer Dermatosen.

Von Privatdozent Dr. Erich Urbacli-Wien 214

Diätformen bei Behandlung des Säuglings- und Kleinkinderekzems. Von

Prof. Marchionini und Dr. Fr. Bòhning-Fqeiborg i. Br 286

Übersicht fiber Arbeiten aus russische $\pi$ Zeitschriften vom 1. juli 1933 bis

1. Juli 1934. Von A. Jon $1 / 8$ wi-Moskau 293

Gesellschaftsberichte. Frühjahrstagung der Vereinigung rheinisch-westfälischer Dermatologen in

Wuppertal-EIberfeld. Sitzung vom 27. Mai 1934224

Royal Society of Medicine. Section of Dermatology. Sitzung vom 15. Febr. 1934170

- $\quad$ Sitzung vom 15. März 1934233

North of England Dermatological Society. Sitzung in Manchester am

9. März 1934234

Société française de Dermatologie et de Syphiligraphie. Sitzungen vom

8. November und 14. Dezember 1933, 11. Januar 1934172

- $\quad$ Sitzungen vom 8. Februar, 3. März, 4. April und 17. Mai 1934 . . 235

Buchbesprechungen 55, 117, 182, 245, 303

Personal ien und Tagesnachrichten 56, 120, 184, 248, 308, 364

Rudolf Mfiller $\dagger \quad 183$

E. Bruusgaard $\dagger \quad 183$

Karl Touton $\uparrow 247$

Sachregister zu Band 66-70 365

Namenregister zu Band 66-70 